\title{
Space Charge Accumulation in XLPE versus Temperature and Water Content
}

\author{
Torbjørn Andersen Ve, Frank Mauseth and Erling Ildstad \\ Department of Electric Power Engineering \\ Norwegian University of Science and Technology (NTNU) \\ Trondheim, Norway
}

\begin{abstract}
The rate of accumulation and amount of space charge is dependent on several factors, including the electrical properties of the insulation material and the temperature of the insulation. Increased water content has been found to increase the conductivity of XLPE up to three times compared to that of dry XLPE, and is therefore also expected to affect space charge accumulation.

This paper presents results of space charge measurements on dry and wet $0.3 \mathrm{~mm}$ thick XLPE insulation at 20,40 and $60{ }^{\circ} \mathrm{C}$. The applied voltage was $10 \mathrm{kV}$, and the measurement period 14 days. Results show that increased water content leads to the formation of heterocharge at the cathode at $20^{\circ} \mathrm{C}$, as opposed to homocharge in case of a dry sample. At higher temperatures, increased water content mainly decreased the magnitude of accumulated charge.

A conductivity-based sectioned model was introduced, and was used to recreate the changes in electric field set up by the accumulated space charge. Comparing the model with the measurements showed that conductivitybased charging was a central mechanism in space charge build-up. At higher temperatures, additional effects not covered by the model were present.
\end{abstract}

\section{Introduction}

New converter technology has made polymer insulation more feasible for HVDC cables again. Space charge accumulation in such cables can strongly enhance the electric field in the insulation. This can result in local fields exceeding the apparent applied field in service. For offshore cables, polymeric or damaged metallic water barriers can lead to water adsorbing into the insulation system. Since water molecules are polar and ionically dissociable, it is expected that high water content will affect the electrical properties of the insulation. The effect of water on space charge has been studied previously [1-3], and it was found that absorbed water can both increase and decrease the accumulation of charge, depending on amount of water in the samples, presence of cross-linking by-products and field strengths. This paper presents results from an experimental investigation of space charge in XLPE samples with low and high water content by the pulsed electroacoustic (PEA) method. The experiments also investigated the effect of temperature. A simplified RC equivalent circuit, based on conductivity differences between the electrode regions and the bulk, was used to model the insulation and estimate the electric fields from the space charge.

\section{Theory}

The transport of charge due to a DC field is given by the conductivity, $\sigma$, through

$$
J=\sigma E
$$

in which $J$ is the current density due to the electric field $E$. Space charge accumulation is directly related to changes in the conductivity and permittivity by Maxwell's equations. While the conductivity of XLPE has been found to vary significantly within the temperature region used in these measurements, the permittivity is mostly constant. A model based on local conductivity variations within the insulation would therefore be a relevant way to analyse the results.

In many cases, space charge has been found to occur mainly close to the insulation surface. In these analyses, it is assumed that conductivity variations between the insulation close to the electrodes and in the bulk is the only factor contributing to space charge accumulation in the model. The insulation is therefore divided into three sections; the anode, bulk and cathode section. The bulk section makes up the centremost $60 \%$ of the insulation, allowing the anode and cathode sections to each make up $20 \%$. These thicknesses were chosen so that an electrode section would contain the space charge peak closest to the electrode.

An RC equivalent, shown in Fig. 1, was used to represent the insulation in the model. The capacitances are calculated from the geometry of the samples, using a constant relative permittivity of 2.3. The resistance values were estimated by assuming that at steady state,

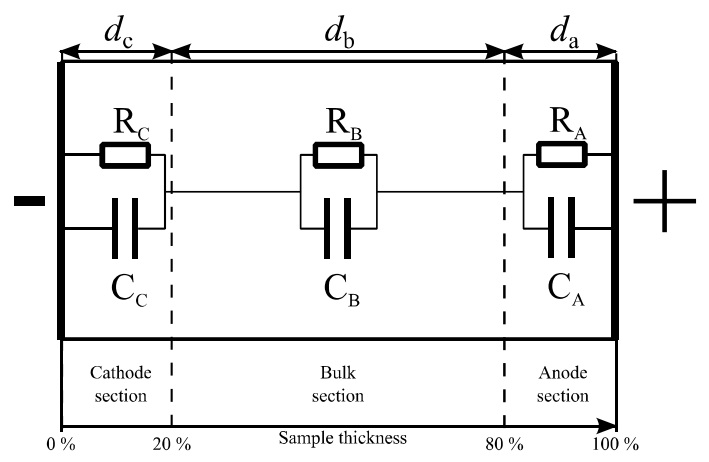

Fig. 1 - Sectioned conductivity model and RC equivalent 
the current densities are constant between sections. Eq. (1) can then be used to find the ratio between the conductivities:

$$
\frac{\sigma_{\mathrm{a}}}{\sigma_{\mathrm{b}}}=\frac{E_{\mathrm{DC}}+E_{\mathrm{b}}}{E_{\mathrm{DC}}+E_{\mathrm{a}}} \quad, \quad \frac{\sigma_{\mathrm{c}}}{\sigma_{\mathrm{b}}}=\frac{E_{\mathrm{DC}}+E_{\mathrm{b}}}{E_{\mathrm{DC}}+E_{\mathrm{c}}}
$$

in which $\sigma_{\mathrm{a}}, \sigma_{\mathrm{b}}$ and $\sigma_{\mathrm{c}}$ is the anode, bulk and cathode section conductivities, respectively, and $E_{\mathrm{a}}, E_{\mathrm{b}}$ and $E_{\mathrm{c}}$ is the electric field from space charge in the anode, bulk and cathode sections, respectively. $E_{\mathrm{DC}}$ is the applied field. The reference conductivities are based on [4]. As the bulk section makes up the majority of the insulation, the conductivity here is set to the value in [4]. As

$$
U_{\mathrm{DC}}=E_{\mathrm{DC}} d
$$

where $U_{\mathrm{DC}}$ is the applied voltage and $d$ the sample thickness, the electric fields in the sections need to fulfil the following relation:

$$
U_{\mathrm{S}}=E_{\mathrm{a}} d_{\mathrm{a}}+E_{\mathrm{b}} d_{b}+E_{\mathrm{c}} d_{\mathrm{c}}=0
$$

where $d_{\mathrm{a}}, d_{\mathrm{b}}$ and $d_{\mathrm{c}}$ is the respective section thicknesses.

\section{Experimental method}

\subsection{Sample preparation}

The samples were XLPE plates with semiconducting polymer electrodes, about $100 \mathrm{~mm}$ in diameter. The upper electrode was $8 \mathrm{~mm}$ in diameter, while the lower electrode covered the entire sample underside. The active area, shown as shaded in Fig. 2, was about 0.3 $\mathrm{mm}$ in thickness.

Prior to measurement, the samples were conditioned to ensure that the water content was at the desired levels. The $20^{\circ} \mathrm{C}$ samples were conditioned at $60{ }^{\circ} \mathrm{C}$, in water for the wet sample and in air for the dry sample, for 4 days. The samples were cooled to $20^{\circ} \mathrm{C}$ before measurement.

For 40 and $60{ }^{\circ} \mathrm{C}$, the wet samples were put in a climate chamber, which was set at $85 \%$ relative humidity. For the conditioning of the dry samples, the humidity system of the chamber was switched off, causing the humidity in the chamber to fall to that of the lab atmosphere. The conditioning time was 2 days at $40^{\circ} \mathrm{C}$, and 1 day at $60{ }^{\circ} \mathrm{C}$.

\subsection{Pulsed electroacoustic test system and measurement procedure}

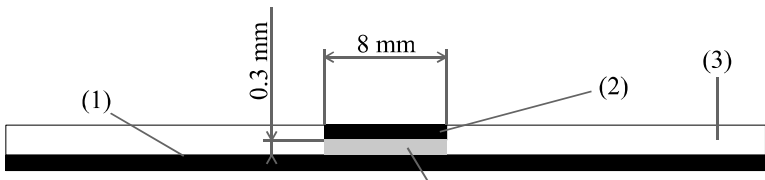

(4)

Fig. 2 - Cross section of sample. (1) Lower semiconductor (2) Upper semiconductor (3) XLPE (4) Active part of insulation (in grey)

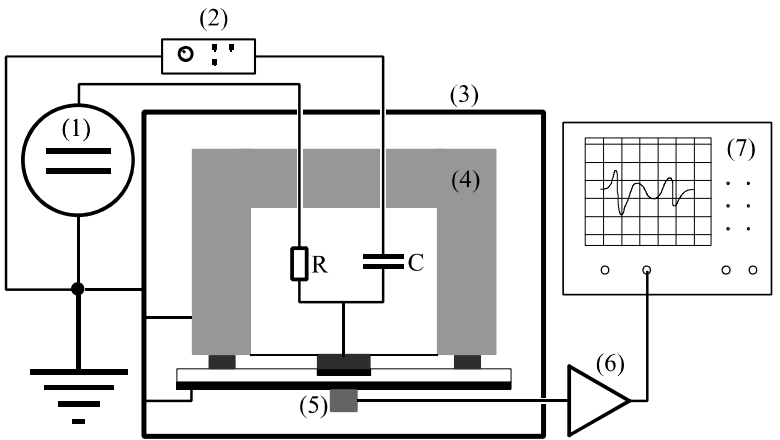

Fig. 3 - Measurement setup (1) HVDC source (2) Impulse generator (3) Climate chamber (4) High voltage electrode (5) Sensor (6) Amplifier (7) Oscilloscope

The test setup, shown in Fig. 3, consisted of a commercial PEA system and a climate chamber. The sample, high voltage electrode and piezoelectric sensor were placed inside the climate chamber. This made it possible to ensure that the samples were kept at the selected temperature and humidity during measurements.

All space charge measurements were performed with DC voltage off, in order to distinguish the space charge signals from the large amounts of induced charge on the electrodes with DC voltage on. Therefore, 30 seconds before each measuring point, the DC voltage was switched off. The signal from the sensor was then recorded, and the voltage switched on again.

\section{Results}

The measured charge distribution for dry and wet samples at $20^{\circ} \mathrm{C}$ is shown in Fig. 4. Homocharge accumulated close to both electrodes in the dry sample. In the wet sample, the amount of homocharge at the anode was about $40 \%$ lower, while heterocharge accumulated at the cathode.

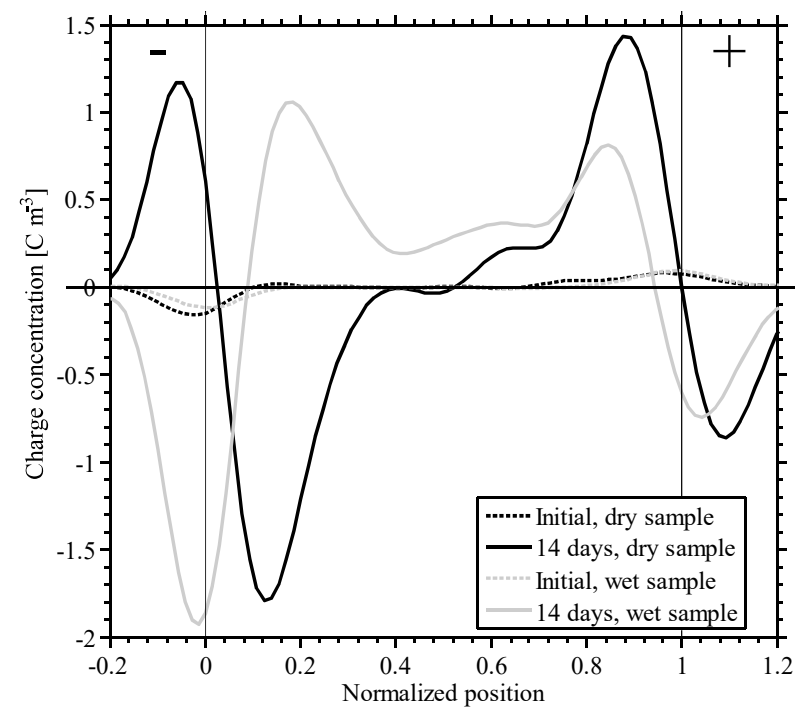

Fig. 4 - Charge distribution at $20^{\circ} \mathrm{C}$, initially and after 14 days, for dry and wet samples 


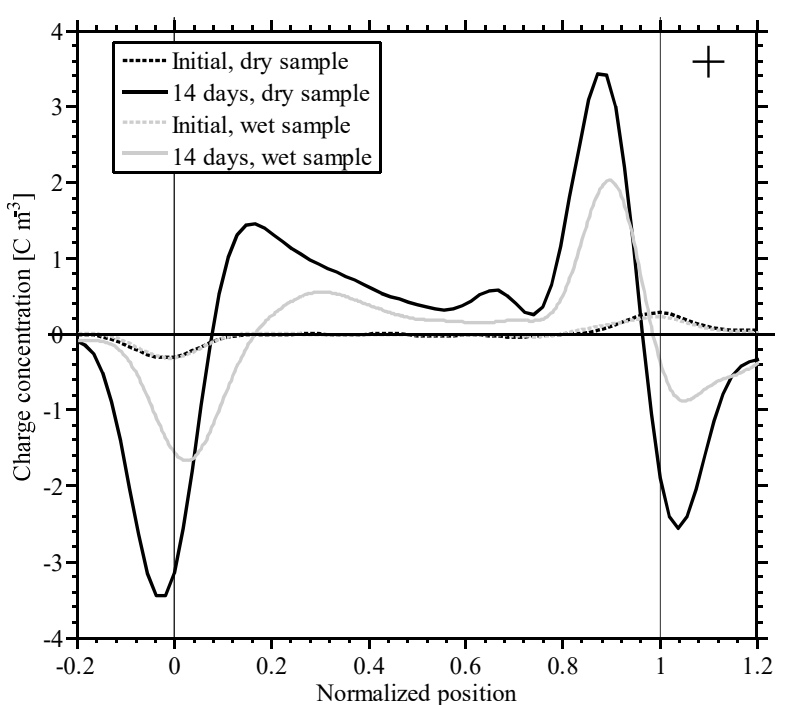

Fig. 5 - Charge distribution at $40{ }^{\circ} \mathrm{C}$, initially and after 14 days, for dry and wet samples

At $40{ }^{\circ} \mathrm{C}$, homocharge accumulated at the anode and heterocharge close to the cathode for both dry and wet samples, see Fig. 5. The wet sample exhibited a lower magnitude of accumulated charge, however. The same was also found in the samples at $60{ }^{\circ} \mathrm{C}$, shown in Fig. 6 , with heterocharge accumulating at the cathode and homocharge at the anode. In general, the magnitude of charge was lower in the wet sample. In addition, while the heterocharge close to the cathode increased from measurement start until after about one hour, it then decreased rapidly for an hour, and kept decreasing for the rest of the test period.

\section{Discussion}

The electric field distribution in the samples can be calculated from the volume integral of the charge density. In Fig. 7, the average electric field from space

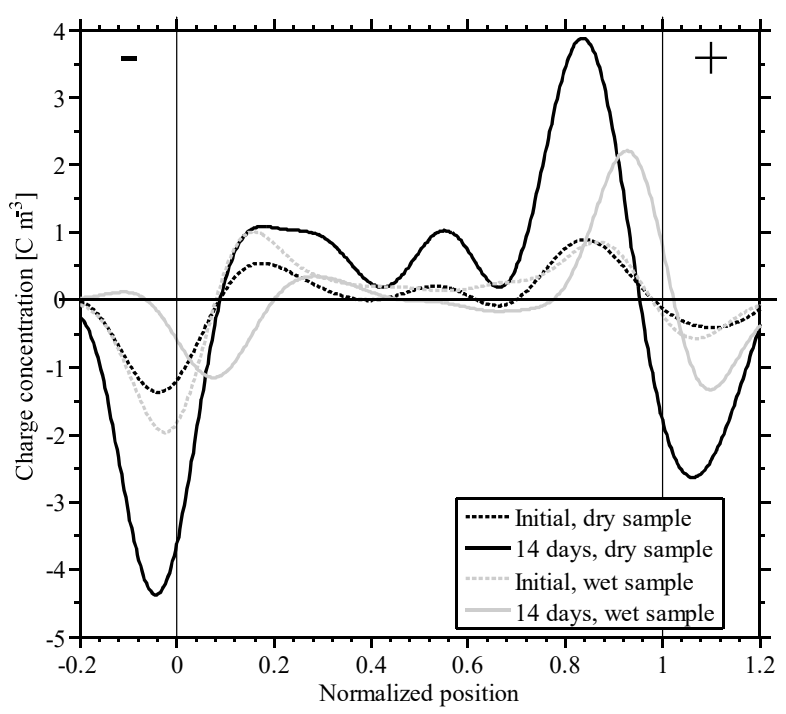

Fig. 6 - Charge distribution at $60{ }^{\circ} \mathrm{C}$, initially and after 14 days, for dry and wet samples

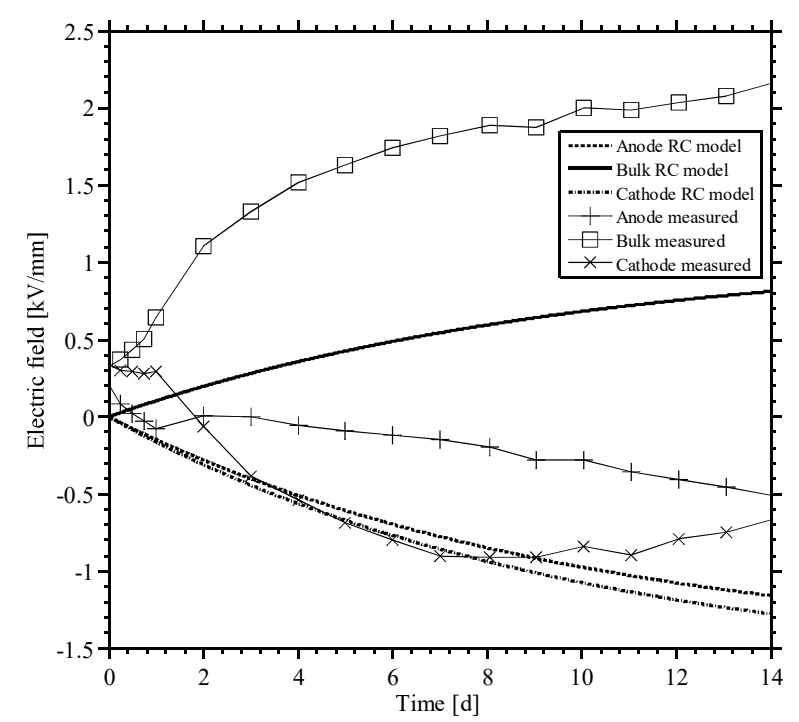

Fig. 7 - Average electric field from space charge, dry sample at $20^{\circ} \mathrm{C}$, and estimated electric field from the RC model.

charge is plotted for the anode, bulk and cathode sections, for the dry sample at $20^{\circ} \mathrm{C}$. In addition, the electric fields in the anode, bulk and cathode sections, as estimated by the RC model, are also plotted. The model correctly predicts that the field is increased in the bulk, and reduced at the electrodes. However, the magnitude of the electric fields in the calculations are lower than the fields in the measurements. This is caused by the estimated fields from the RC model fulfilling (2), whereas the fields in the measurements results in a positive $U_{\mathrm{s}}$. Contributing factors to this discrepancy is the choice of electrode placements and section thicknesses, and the numerical procedures used in the treatment of the measured data. In the numerical procedures, the parts of the signal representing the electrodes are selected. As this selection is the basis of the rest of the calculations, a small deviation here would

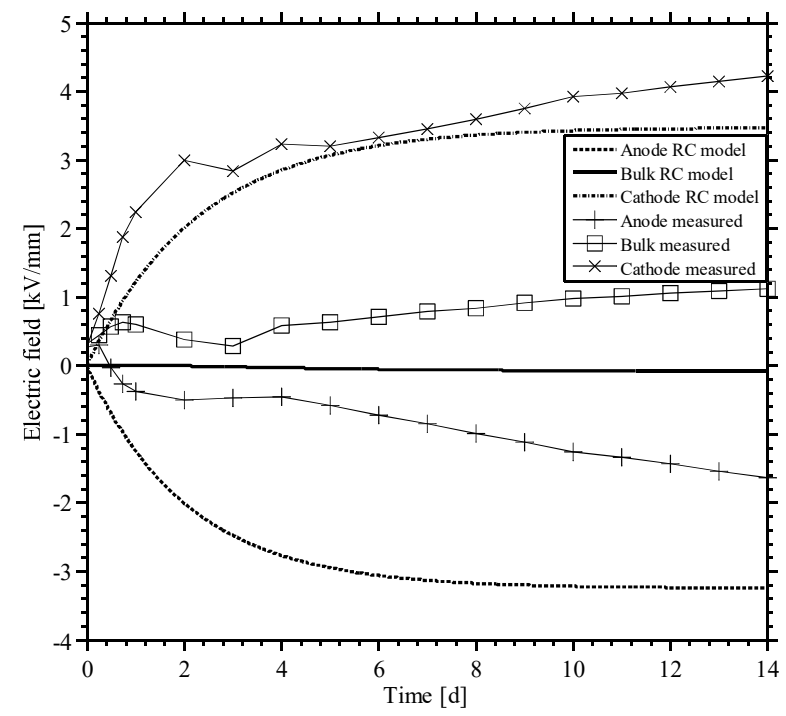

Fig. 8 - Average electric field from space charge, wet sample at $20^{\circ} \mathrm{C}$, and estimated electric field from the RC model. 


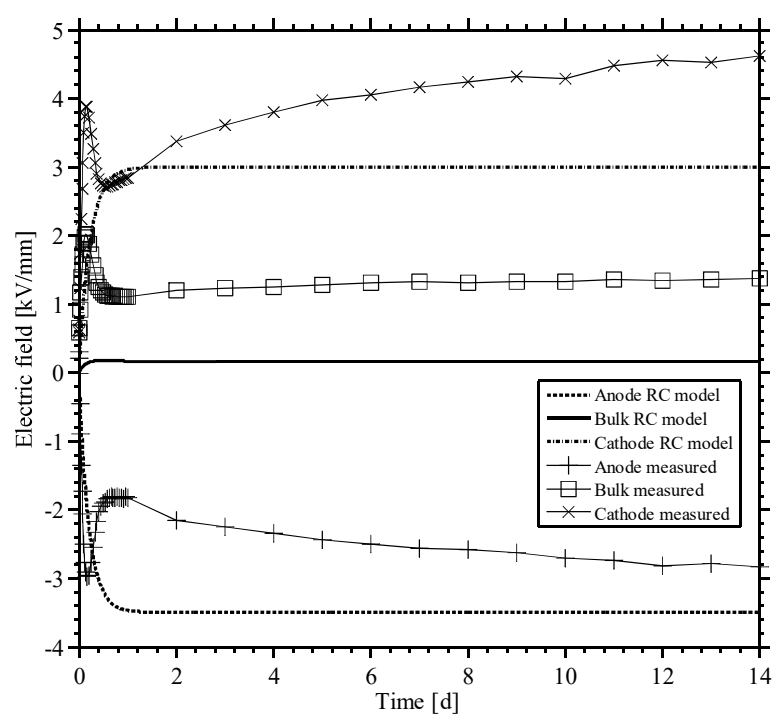

Fig. 9 - Average electric field from space charge, dry sample at $40{ }^{\circ} \mathrm{C}$, and estimated electric field from the RC model.

result in affect the calculated charge distribution. As the electric field is calculated by the integral of the charge distribution, the effect on this could be significant.

The electrode positions are placed at the electrode peaks in space charge measurements with DC voltage on. The section thicknesses, which are used to calculate the average fields in the measurements and the values of resistances and capacitances in the $\mathrm{RC}$ model, are dependent on these. The results are therefore sensitive to the choice of these positions, particularly since the where the broadness and placement of the measurement peaks vary between measurement series, and sometimes also within a series.

Looking at the conductivities used, the time constant from the $20^{\circ} \mathrm{C}$ dry sample in [4] is about 10 days, while the apparent time constant in the measurements, calculated from the bulk electric field, is about 5 days. A halving of the time constant is equivalent to a doubling of the conductivity. Using a higher conductivity in the RC model would therefore be expected to yield results showing a time development closer to what was found in the measured results.

In Fig. 8, showing the electric fields from measurements and the $\mathrm{RC}$ model for the $20^{\circ} \mathrm{C}$ wet sample, the relative differences between the electric fields in the various sections are similar for the measured and calculated results. While the calculated cathode field seems to be reasonably close to what was measured, the field at the anode and in the bulk lie lower than what was found in the measurements.

At 40 and $60{ }^{\circ} \mathrm{C}$, the estimated fields from the $\mathrm{RC}$ model follow the same trends as the measurements. At these temperatures, the measurements display additional effects not found in the electric fields from the RC model. An example of this can be seen in Fig. 9, which shows the results for a dry sample at $40{ }^{\circ} \mathrm{C}$. A rapid increase in field occurs during the first 4 hours of the measurement, followed by a rapid decrease for about 9 hours. This is not found in the estimated fields from the
RC model, which predicts charging towards a steadystate value.

\section{Conclusions}

Space charge accumulation at 20,40 and $60{ }^{\circ} \mathrm{C}$ in XLPE samples with high and low water content was measured. A conductivity-based sectioned RC equivalent was used to model the changes in electric field in the samples. Similarities between the model and the measurements suggest that conductivity based charging may be a central mechanism in space charge build-up. At higher temperatures, evidence of additional mechanisms not covered by the model was found.

\section{Acknowledgment}

This work is part of the Norwegian Renergi R\&D project "High Voltage AC and DC Subsea Cables for Offshore Wind Farms and Transmission Grids" funded by the Research Council of Norway and the industrial partners Nexans Norway AS, EDF R\&D (France), Statoil ASA, Statnett SF, Statkraft SF and Borealis (Sweden).

\section{References}

[1] H. Miyata, A. Yokoyama, T. Takahashi, and S. Yamamaoto, "Effect of water on the space charge formation in XLPE," in 1996 IEEE International Symposium on Electrical Insulation, Montreal, Canada, 1996, pp. 670673.

[2] Y. Sekii, T. Ohbayashi, T. Uchimura, T. Hukuyama, and T. Maeno, "A study on the space charge formation in XLPE," in Electrical Insulation and Dielectric Phenomena, 2001 Annual Report. Conference on, 2001, pp. 469472.

[3] G. C. Montanari and F. Palmieri, "The effect of humidity on space charge accumulation in XLPE," presented at the 2003 Annual report conference on electrical insulation and dielectric phenomena, 2003.

[4] T. A. Ve, F. Mauseth, and E. Ildstad, "Effect of water content on the conductivity of XLPE insulation," in Electrical Insulation and Dielectric Phenomena (CEIDP), 2012 Annual Report Conference on, 2012, pp. 649-653. 